\title{
A Deoxyribozyme That Forms a Three-Helix-Junction Complex With Its RNA Substrates and Has General RNA Branch-Forming Activity
}

\author{
Rebecca L. Coppins and Scott K. Silverman*
}

Department of Chemistry, University of Illinois at Urbana-Champaign, 600 S. Mathews Ave., Urbana, Illinois 61801

\section{Demonstrating Watson-Crick covariation for the RNA:DNA nucleotides within the P4 duplex}

The data in manuscript Figure 5D show that Watson-Crick matched combinations of R substrate and deoxyribozyme nucleotides in paired region P4 all provide substantial ligation rate and yield, although the transv-2 combination has a relatively low ligation rate. Here we examined more comprehensively the RNA:DNA combinations for these three nucleotide pairs in P4 (Figure X1). The data are entirely consistent with straightforward Watson-Crick base pairing between these RNA and DNA nucleotides. This experiment is related to that shown in Figure 4b of ref. 11, which examined paired regions $\mathrm{P} 1+\mathrm{P} 2$.

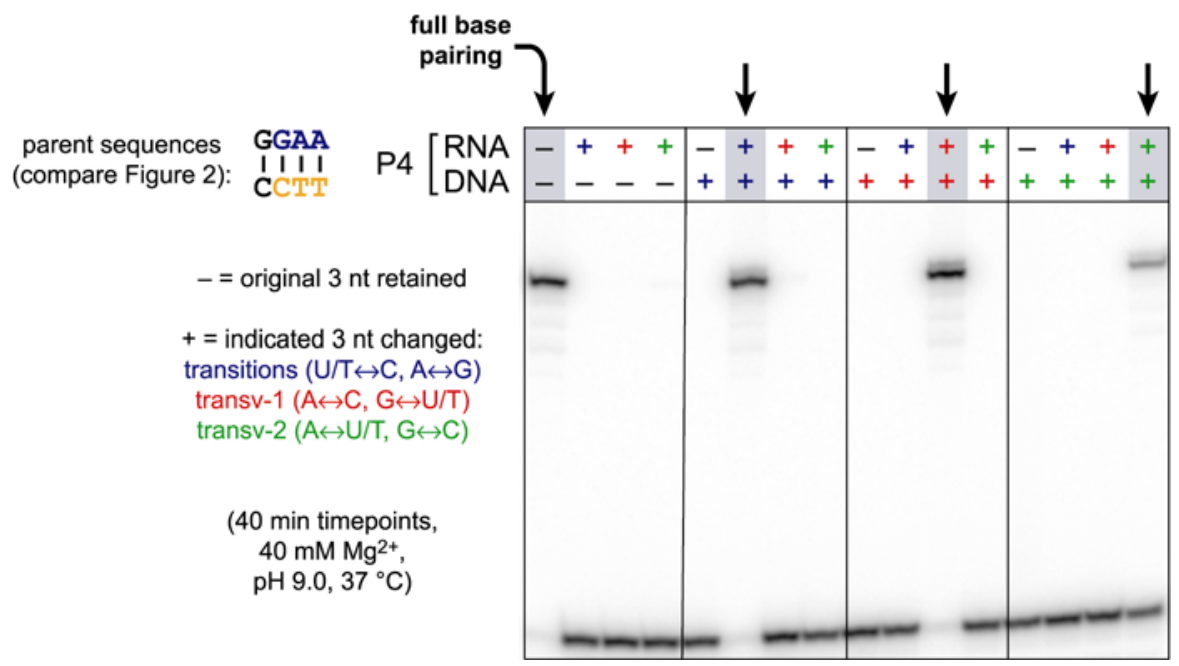

Figure X1. Comprehensively demonstrating the existence of Watson-Crick base-paired duplex region P4 by testing matched and mismatched RNA:DNA combinations. Incubation conditions were $50 \mathrm{mM} \mathrm{CHES,} \mathrm{pH} \mathrm{9.0,} 150$ mM NaCl, 2 mM KCl, 40 $\mathrm{mM} \mathrm{MgCl}$, and $37^{\circ} \mathrm{C}$. The parent $\mathrm{P} 4$ sequence is shown at the left; for the various assays, three (of four) nucleotides on the right side of each strand were changed or left unchanged as indicated. In all cases, the 5'-terminal RNA nucleotide of the R substrate was 5'-triphosphate-G, and its DNA nucleotide counterpart was C. The lanes marked with bold arrows correspond to single 40-min timepoints for the matched combinations. Full kinetic data for the matched combinations from an independent analogous experiment are shown in manuscript Figure 5D. 
Examining requirement for the branch-site 5'-activated RNA nucleotide and its DNA counterpart in P4

Figure X1 above shows experimental examination of the three $\mathrm{P} 4$ nucleotides that do not include the 5'-terminal guanosine of the R substrate. Examination of the 5'-terminal guanosine RNA nucleotide and its DNA counterpart are presented here. Figure X2 shows data obtained for substrates that retain a 5'triphosphate-G on the right-hand RNA substrate along with all possible DNA nucleotides as its counterpart. The G RNA + C DNA Watson-Crick combination provides the most favorable ligation rate. However, G RNA + A DNA is more favorable than G RNA + T DNA, suggesting that a simple base-pairing requirement is not the only consideration. Nevertheless, for a 5'-G in the RNA, the Watson-Crick DNA match (C) offers the best ligation rate and yield.

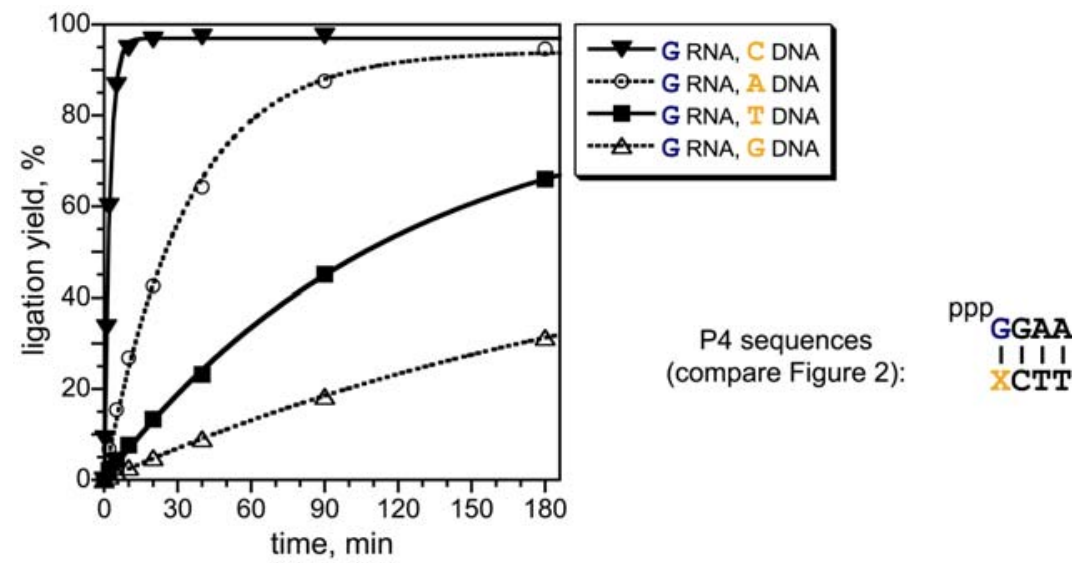

Figure X2. Testing the importance of a Watson-Crick base pair at the 5'-terminal nucleotide combination of the P4 duplex. In all cases, the 5'-terminal guanosine nucleotide of the R substrate was 5'-triphosphorylated. Assays were performed under the incubation conditions described in Figure X1. $k_{\text {rel }}$ values (top to bottom): 1.0, 0.68, 0.019, and 0.0093.

In a separate assay shown in Figure X3, the 5'-terminal nucleotide of the right-hand RNA substrate was changed from $\mathrm{G} \rightarrow \mathrm{A}$, and all possible DNA nucleotides were tested as its counterpart. This is similar to Figure X2, which used a G in the right-hand RNA substrate. The right-hand substrate was 5'adenylated for Figure X3. Again, the data demonstrate that a Watson-Crick RNA:DNA base pair is optimal between the 5'-terminal nucleotide of the R substrate and its DNA counterpart.

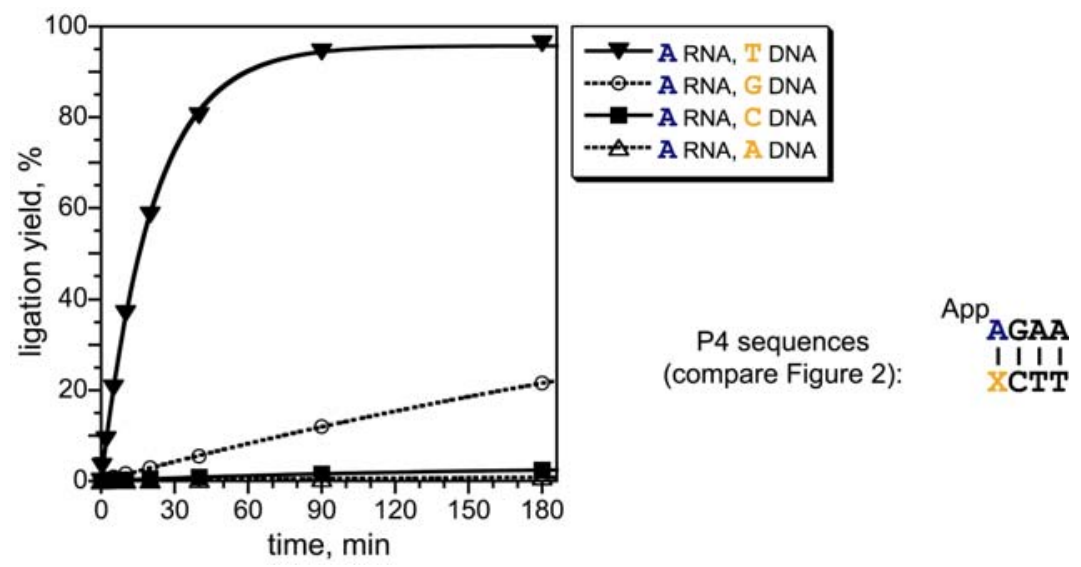

Figure X3. Testing the importance of a Watson-Crick base pair at the 5'-terminal nucleotide combination of the P4 duplex. In all cases, the 5'-terminal adenosine nucleotide of the $\mathrm{R}$ substrate was 5'-adenylated. Assays were performed under the incubation conditions described in Figure X1. $k_{\text {rel }}$ values: T DNA 1.0, G DNA 0.050. 